Original Research Paper

\title{
A Review of Recent Results on 3D Effects
}

\author{
${ }^{1}$ Filippo Berto, ${ }^{2}$ Relly Victoria V. Petrescu and ${ }^{2}$ Florian Ion T. Petrescu \\ ${ }^{I}$ Department of Engineering Design and Materials, NTNU, Trondheim, Norway \\ ${ }^{2}$ Bucharest Polytechnic University, 313 Splaiul Independentei, Bucharest, Romania
}

Article history

Received: 16-10-2016

Revised: 23-12-2016

Accepted: 27-12-2016

Corresponding Author:

Filippo Berto

Department of Engineering

Design and Materials, NTNU,

Trondheim, Norway

Email: berto@gest.unipd.it

\begin{abstract}
Three dimensions or three-dimensional or 3D are expressions that characterize the space around us, as perceived by our vision, in terms of width, height and depth. The term "3D" is also (and improperly) used to refer to the representation in synthetic (digital) images, the relief of stereoscopic images or other images in relief and sometimes even the simple stereophonic effect, which Can only render 2D (it is therefore only the calculation of the projected projections, the shadows, the renderings of materials). In mathematics, this notion corresponds to Euclidean geometry in space. The space is marked by three orthogonal axes, contrary to the plane composed of two dimensions. Since its origins and long before the appearance of colour, cinema has exploited stereoscopy, mainly through the processes of anaglyphs. During the $1950 \mathrm{~s}$, the Hollywood studios exploited the polarized filter system and then, from the $2000 \mathrm{~s}$ onwards, with the adoption of digital formats, the rooms also equipped the electronic principle with alternating shutter ("active" glasses). Despite some anaglyphic experiments since the 1930 and $1950 \mathrm{~s}$, the commercial success of 3D television became a reality from the end of the $1990 \mathrm{~s}$ to a real industrial development from the year 2000. Two principles and devices for Stereoscopic effects are commercialized: Of the "active" type with electronic bezels or of the "passive" type with polarized filter goggles. More complex and costly to industrialize in particular with regard to large screens, auto stereoscopy (relief effect without bezel) was introduced with Alioscopy since the late $1990 \mathrm{~s}$, on the lenticular principle. Stereoscopic videoprojection intended for the general public requires electronic "active" glasses or a special screen (metallised or offering some refraction of light) associated with "passive" glasses with polarized filters. ThreeDimensional (3D) effects close to sharp corners of a hole in a plate with finite thickness are investigated in the present contribution. The results from detailed 3D finite element model are analyzed to investigate the stress intensity of various fracture modes caused by the presence of a finite thickness. The results expressed in terms of stressed are compared with some recent equations. The comparison between numerical and theoretical results shows a sound agreement.
\end{abstract}

Keywords: 3d Effects, Finite Element Analysis, Hole, Linear Elasticity

\section{Introduction}

Three dimensions or three-dimensional or 3D are expressions that characterize the space around us, as perceived by our vision, in terms of width, height and depth. The term "3D" is also (and improperly) used to refer to the representation in synthetic (digital) images, the relief of stereoscopic images or other images in relief and sometimes even the simple stereophonic effect, which Can only render 2D (it is therefore only the calculation of the projected projections, the shadows, the renderings of materials). In mathematics, this notion corresponds to Euclidean geometry in space. The space is marked by three orthogonal axes, contrary to the plane composed of two dimensions. The three geometric dimensions are: 
- The width (left/right) of axis x, or abscissa

- The height (up/down) of axis y, or dimension

- The depth (front/back) of $\mathrm{z}$-axis, or ordinate, or depth

In mathematics, the notion of dimension is more extensive and not limited to geometry.

In computer science, three-dimensional models (figures or synthetic images) require computations without particular complexity, but extremely numerous.

They can be represented either by perspectives of various directions on a two-dimensional screen (which makes the term "3D" improper, a screen having only two dimensions), or on devices of the embossed film type or spectacles Liquid crystals to see a different image with each eye.

Since the late $1990 \mathrm{~s}$, most computers have an add-on processing unit dedicated to this type of calculations (graphics processor or GPU). Several software, including Blender, to create these 3D models with conventional computers, which is then referred to as a $3 \mathrm{D}$ image synthesis.

Several techniques can be used to print images that can give an impression of depth:

Stereoscopic image requiring the wearing of specific, bi-colored or polarizing spectacles, enabling the brain to reconstitute stereoscopic vision.

Holography: The support is a transparent film for a transmission image or a silver film for a reflection image and contains an interference pattern obtained with a laser. The diffraction on this figure, similar to a diffraction grating, creates a volumetric image visible to the naked eye but does not allow a colour rendering.

Other techniques allow or will allow do this without special glasses.

Imaging lenticular (postcard type or gadgets) usually printed (or laminated) on grooved support on the spectator side.

In Germany, a technique was developed in Germany combining a particular mode of printing and illumination producing large $3 \mathrm{D}$ images.

The prototype realized in 2010 measured 1 square meter (format A0) but the group estimates to be able to reach a panel of five meters. The image previously modified by computer is printed on a network of hundreds of thousands of lenses which return it in a different way according to the point of view. Several thousand points of view are possible.

Since its origins and long before the appearance of colour, cinema has exploited stereoscopy, mainly through the processes of anaglyphs. During the 1950s, the Hollywood studios exploited the polarized filter system and then, from the $2000 \mathrm{~s}$ onwards, with the adoption of digital formats, the rooms also equipped the electronic principle with alternating shutter ("active" glasses).
Despite some anaglyphic experiments since the 1930 and $1950 \mathrm{~s}$, the commercial success of 3D television became a reality from the end of the $1990 \mathrm{~s}$ to a real industrial development from the year 2000. Two principles and devices for Stereoscopic effects are commercialized: Of the "active" type with electronic bezels or of the "passive" type with polarized filter goggles. More complex and costly to industrialize in particular with regard to large screens, autostereoscopy (relief effect without bezel) was introduced with Alioscopy since the late $1990 \mathrm{~s}$, on the lenticular principle.

Stereoscopic videoprojection intended for the general public requires electronic "active" glasses or a special screen (metallised or offering some refraction of light) associated with "passive" glasses with polarized filters.

$3 \mathrm{D}$ printing or three-dimensional printing is the common terms used to talk about additive manufacturing processes. Initially, due to their original defects, these processes have been developed for rapid prototyping, but are increasingly used for the manufacture of functional parts. Although others exist, here are some technologies that co-exist: Fused Deposition Modelling (FDM), SLA (Stereo-lithography: UV light solidifies a layer of liquid plastic) and selective laser sintering (A laser agglomerates a layer of powder).

Three-dimensional printing makes it possible to produce a real object: An operator draws the object on a screen using a Computer Aided Design (CAD) tool. The $3 \mathrm{D}$ file thus obtained is sent to specific software which cuts it into slices and sends it to the printer which deposits or solidifies the material layer by layer until obtaining the final part. The principle is therefore quite similar to that of a conventional 2D printer: The nozzles used, which deposit material, are the same as those used in office printers. It is the stacking of these layers that creates a volume.

In 2013, this technique is limited to the use of materials not conducive to intensive use and therefore only produces prototypes, sometimes life-size, but the use that will be made of it in the future remains a subject of research and debate. The dental industry and luxury Jewellery however already use 3D printing successfully for the realization of final parts. The aerospace, automotive and film industries are also already using 3D printing successfully for finished parts. Some users of personal 3D printers or online 3D printing services also use 3D printed objects on a daily basis. Started in January 2013, the European Space Agency's Amaze project aims to enable industrial 3D printing of aerospace parts and other high-stress areas. Furthermore, in Amsterdam, a 3D printed bridge should be commissioned in 2017 to link two banks of a 6.5 meter wide canal.

Chris Anderson, American writer and journalist, author of Makers: The New Industrial Revolution, sees 
the advent of three-dimensional printing as a third industrial revolution. Barack Obama, President of the United States, indicated his willingness, in his State of the Union address in February 2013, for America to invest in the creation of $3 \mathrm{D}$ printing Stimulate innovation and create jobs. Its development could relocate production in rich countries, given that now the workforce would have become obsolete.

In France in 2013, the Ministry of Productive Recovery, supported by a call for projects, 14 fablabs (or additive laboratories), using 3D printing machines.

The democratization of three-dimensional printing gives rise to fears of new modes of illegal reproduction. It could lead to an adaptation of intellectual property law.

In November 2010, Michael Weinberg wrote on PublicKnowledge.org. that "The time will come (...) quickly where the industries in place that will be affected will demand new restrictive laws for $3 \mathrm{D}$ printing. If the community awaits this day to organize, it will be too late. Instead it must strive to educate decision makers and the public to the tremendous potential of $3 \mathrm{D}$ printing. Thus, when incumbent industries disdain 3D impression as a pastime of pirates or outlaws, their statements will fall into ears too wise to destroy this brand new novelty."

Three-dimensional printing was initially industrial, but also works on the free/open source model. An author of Framablog writes "think indeed of a world where the four freedoms of the software also apply to domestic objects: Freedom of use, study, improvement and diffusion. Are we not then really in conditions that allow us to free ourselves from a certain economic and financial logic of which we could only notice helpless damage ever more numerous?"

For example, blogger Todd Blatt received a demand from Paramount for the materialization of a cube similar to that of the Super 8 film on Shape ways (database of 3D digital files). In August 2013, Shapeways was also put on notice by the Japanese company Square Enix, editor of the game Final Fantasy, following the posting and sale of figurines representing the heroes of the game on their site.

In 2013, MP Cornut-Gentille questioned the Minister of Productive Recovery (Arnaud Montebourg) at the National Assembly: "The proliferation of download sites of this type of file is to be feared in the years to come; In the long term, it could lead to effects as detrimental to industry as those currently experienced in the music and cinema sectors", fearing reproductions made "without any property rights and at a lower cost" Private plans are recovered. The Member wishes to know whether there are 'arrangements' for regulating and supervising the market for three-dimensional printing (e.g., by deploying digital locks (DRM)).

The three-dimensional printer first came from science fiction (Arthur C. Clarke evoked a "machine to replicate" in the $1960 \mathrm{~s}$, a machine that would replicate objects as printed books, which would have a profoundly positive effect On society: "Humanity will adapt as in the past") or comic strip (in 1972, in the cartoon Tintin and Shark Lake, Professor Tournesol invented a three-dimensional photocopier immediately coveted by Rastapopoulos to make Fakes by duplicating works of art stolen from large museums).

Then in the last quarter of the twentieth century some industries developed it and used it (for prototyping especially).

On July 16, 1984, the first patent on "additive manufacture" was filed by Jean-Claude André, Olivier de Witte and Alain le Méhauté for the company CILAS ALCATEL. Two weeks later, the American Chuck Hull breaches the technique of stereo lithography (SLA for Stereo Lithography Apparatus). This patent is at the origin of the extension of the ".stl print file" and of 3D Systems, a giant manufacturer of $3 \mathrm{D}$ printers. In late 1988, it launched the first 3D printer, the SLA-250.

In 1995, DMLS metallic 3D printing technology was introduced for Direct Metal Laser Sintering. In 2003 the process of pasting sheets of A4 paper, 3DPP, for 3D Paper Printing appears.

In 2005, the first colour printer (company $\mathrm{Z}$ Corporation) was born, using four-color printing as conventional printers and pigments bonded by glue to a mineral material.

The year 2006 saw the birth of the project RepRap, the first open source 3D printer project, by Dr. Adrian Browyer (then professor of mechanical engineering at the University of Bath). It paves the way for future home printers, since it consists in being able to build by itself a $3 \mathrm{~d}$ printer in technology of deposit of molten wire. It gives birth to the culture maker.

It is in 2011 that appears 3D printing food, based on sugar or chocolate.

Many observers believe that these technologies will play an important role in the new form of production. Jeremy Rifkin believes that it could be one of the elements of his concept of the third industrial revolution.

The precision of the printing and the typologies of materials are likely to increase in the future as technical advances.

The sand, which has long been used in casting, seems to have been first tested for 3D printing in 1999 in Germany by the start-up Generis (which in 2003 was split into ProMetal, devoted to sand printing and Voxeljet, Specializing in lost-wax casting). More recently (2012-2013), artistic and/or technical experiments have used machines constructing objects or decorations in sand, sometimes of large size. This is the case for example: Architectural decors printed by architects Michael Hansmeyer and Benjamin Dillenburger, realized with the Architecture Department 
of ETH Zurich. The 3D machine produced large sandbased objects shaped from algorithms designed to produce complex and decorative shapes 20, which were exhibited in France at the FRAC in Orléans in 2014.

"Sculptures" or utilitarian objects constructed by a computer-controlled "stone spray robot" capable of printing in several directions at the same time (in two planes, vertical and horizontal) to produce complex shapes (Furniture, walls, sculptures built of sand agglomerated by an adhesive (LEEED certified binder), with a power supply that is a photovoltaic panel. This robot was produced by Shergill, Anna Kulik and Petr Novikov, supervised by Jordi Portell, Marta Male Alemany and Miquel Iloveras of the Institute for Advanced Architecture of Catalonia (IAAC).

Objects in molten sand. The fusion being here produced by concentrating a beam of sunlight on a renewed layer of desert sand; Markus Kayser has produced a small, very simple prototype, successfully tested in the Sahara desert, using a solar sinter project concentrating the heat of the sun on sand added in the machine layer by layer, on the basis of a numerical model.

Large objects may soon also be produced by Contour crafting technology: Prof. Behrokh Khoshnevis, with the University of Southern California and NASA funding and the Cal-Earth Institute will test in 2014 a "3D printer Giant "with a project to build a house in 24 hours.

The printer here is a robot that projects concrete according to a plan stored in the computer that controls it. Such robots could construct, for all or part of civilian and military buildings, landing strips, roads, sheds or anti-radiation walls as well as possibly habitable structures on the moon, Or other extraterrestrial environments. Tests are done in a laboratory located in the Nasa Desert (D-RATS). This process is or has been tested on a small scale (project "house of the future/Urban initiative policy" (2004)) and has been considered by industrialists for several years.

Robots capable of printing three-dimensional structures can, for example, construct a self-supporting bridge (of modest size) without the need for scaffolding and by "printing" their own support structures that can become pieces of architecture at As his plan materializes. A first project focused on the use of sand as a base material and mid-2015, a Dutch start-up has announced plans to test (mid-2017) the construction of a pedestrian bridge 7 meters above sea level, A canal in Amsterdam, based on the so-called "out of box" method. In this case the robots will build the bridge by projecting small amounts of molten steel via 6-axis moving arms with a specially developed welding gas (by liquid air).

In 2012, the global 3D printing market reached $\$ 2.2$ billion with an annual growth of $30 \%$.

In 2013, it is easy to manage materials with the following characteristics:
Electrical and insulating conductors, electric resistors;

Transparent, translucent or opaque;

Rigid or flexible, elastic or brittle;

Pasty, hard, abrasive;

Colored (all colors, including phosphorescent, UV reagents...);

Magnetically inert.

Magnetically temporary (by introducing iron filings into a cavity or a metal for example; A subsequent magnetization is used to manage the final magnetization).

In 2013 , it is not possible to easily manage materials with the following characteristics:

Neutral, negative and positive semiconductors: see dedicated article: doping (semiconductor);

Preloaded;

Gas.

The textiles are usually assembled separately and attached to the final objects.

This makes it impossible to transistors, electronics, computers, photovoltaic panels, flexible leaf switches.

It is easier to build the parts separately and then assemble them, but it is often possible to print the already finished objects with the assembly already done.

Note that Microsoft filed in September 2013 a patent made public that would aim to push the limits described above: Indeed, the company envisages 3D printers capable of elaborating electronic objects by providing as "consumables" cartridges of electronic components (chips, led, processors, etc.).

Two-dimensional solutions based on theory of elasticity are widely employed because they could serve as a starting point for different engineering solutions, standards in force and criteria for the fracture assessment. In terms of expensiveness, 2D models, based on simplified plane stress or plane strain hypothesis, are very efficient, easy to use if compared with the corresponding 3D solutions. However, to well describe the thickness effect of a real structure, alternative methods have to be employed.

3D effects were investigated for cracks in plates with a finite thickness by employing different analytical and numerical approaches (Nakamura and Parks, 1989; Jin and Batra, 1997; Heyder et al., 2005). Dealing with $3 \mathrm{D}$ stress distribution in presence of zero notch radius notches characterized by different notch opening angles an advanced theory based on generalised plane strain conditions (Kane and Mindlin, 1956) is investigated in (Kotousov, 2005; 2007). The mode in mode II loading was named 'the out-of-plane mode, or Mode O', differentiating it from Mode 3. It has been also widely proofed that mode $\mathrm{O}$ is caused by $3 \mathrm{D}$ effects 
connected to Poisson's ratio and described by the same equation valid to describe Mode III.

The coupling of the loading modes dealing with sharp notches was investigated in (Lazzarin and Zappalorto, 2012). It has been shown that the governing equations of $3 \mathrm{D}$ elasticity can be simplified to a biharmonic equation. The former provides the solution of the $2 \mathrm{~d}$ problem, while the latter provides the anti-plane solution. Satisfying the equations simultaneously the coupled interaction between different modes can be easily explained. The objective of the present investigation is to apply the available theory in (Lazzarin and Zappalorto, 2012) for investigating the stress distribution of sharp notches in plates with a finite thickness under different constraint conditions.

\section{Approach}

\section{D Stress Distribution}

A recent approach for the investigation of $3 \mathrm{D}$ problems was proposed successfully by Lazzarin and Zappalorto (2012) by assuming a generalized plane strain condition (Kane and Mindlin, 1956) for displacements accordingly to the following equations:

$u_{x}=u(x, y) u_{y}=v(x, y) u_{z}=b z \times w(x, y)$

In Equation $1 \mathrm{~b}$ is a constant. Accordingly to this assumption the normal strains $\varepsilon_{x x}, \varepsilon_{y y}, \varepsilon_{z z}$ as well as $\gamma_{x y}$ are not a function of $z$, while on the other hand, the other components, i.e., $\gamma_{y z}$ and $\gamma_{x z}$, are a function of $z$.

The following equations are as follows:

$$
\begin{aligned}
& \varepsilon_{x x}=\frac{\partial u}{\partial x} ; \varepsilon_{y y}=\frac{\partial v}{\partial y} ; \varepsilon_{z z}=b w \\
& \gamma_{x y}=\frac{\partial u}{\partial y}+\frac{\partial v}{\partial x} ; \gamma_{y z}=b z \frac{\partial w}{\partial y} ; \gamma_{x z}=b z \frac{\partial w}{\partial x}
\end{aligned}
$$

By using the stress-strain relationship, the stress components $\sigma_{x x}, \sigma_{y y}, \tau_{x y}$ and $\sigma_{z z}$ are independent of $z$, whereas the mode II stress components depend on $z$, according to the expressions:

$$
\begin{aligned}
& \sigma_{z z}=\frac{E}{(1-2 v)(1+v)}\left[(1-v) \varepsilon_{z z}+v\left(\varepsilon_{x x}+\varepsilon_{y y}\right)\right] \\
& =\frac{E}{(1-2 v)(1+v)}\left[(1-v) b v+v\left(\frac{\partial u}{\partial x}+\frac{\partial v}{\partial y}\right)\right] \\
& \tau_{y z}=G \gamma_{y z}=G b z \frac{\partial w}{\partial y} ; \tau_{x z}=G \gamma_{x z}=G b z \frac{\partial w}{\partial x}
\end{aligned}
$$

By employing the conditions in $\mathrm{z}$ direction, it can be obtained: $\nabla^{2} w=0$

where, $\nabla^{2}$ denotes the two-dimensional Laplacian operator.

By considering the equilibrium condition in $x$ and $y$ directions the following equation can be derived:

$\frac{\partial^{2} \sigma_{x x}}{\partial x^{2}}+\frac{\partial^{2} \sigma_{y y}}{\partial y^{2}}+2 \frac{\partial^{2} \tau_{x y}}{\partial y \partial x}=0$

Due to the fact that the stress components $\sigma_{x x}, \sigma_{y y}, \tau_{x y}$ and $\sigma_{z z}$ do not depend on $z$, Equation 5 is automatically satisfied by the classic Airy stress function $\phi(x, y)$ :

$\sigma_{x x} \frac{\partial^{2} \phi}{\partial y^{2}} ; \sigma_{y y} \frac{\partial^{2} \phi}{\partial x^{2}} ; \tau_{x y}=-\frac{\partial^{2} \phi}{\partial x \partial y}$

Now, by using the Lame's equations for stresses and strains the following equation can be easily obtained (Lazzarin and Zappalorto, 2012):

$\nabla^{4} \phi=v \nabla^{2} \sigma_{z z}=0$

Accordingly to this frame any $3 \mathrm{D}$ problem can be separated into a bi-harmonic problem and an harmonic problem. The final system can be written as follows:

$\left\{\begin{array}{l}\nabla^{4} \phi=0 \\ \nabla^{2} w=0\end{array}\right.$

where, $\phi$ and $w$ are properly defined by Equation 3 and 6 , respectively. Both Equation $8 \mathrm{a}$ and $8 \mathrm{~b}$ need to be satisfied simultaneously.

\section{Comparisons with FE Models}

In this special section, a 3D model with an inclined diamond hole in a finite thickness plate in varying load conditions is taken into consideration. In order to demonstrate the degree of accuracy of the theory developed in (Lazzarin and Zappalorto, 2012), the analytical frame of the theory is explained first and then verified the Finite Element 3D models (FE), being under torsion and tension loading conditions respectively.

\section{The Spatial Diamond Hole Problem}

A hole in a $3 \mathrm{~d}$ plate under tension and torsion is considered in this section. The angle of inclination has been set equal to $22.5^{\circ}$, Fig. 1. Such an inclination provokes local in-plane mixed mode stresses (mode I + mode II). They can be solved by using Williams' solution for notches, according to Equation 8, where the function $\phi$ can be expressed as (Lazzarin and Zappalorto, 2012; Williams, 1952): 


$$
\begin{aligned}
& \phi=r^{\lambda_{1}+1}\left[A_{s} \cos \left(\left(\lambda_{1}+1\right) \theta\right)+B_{s} \cos \left(\left(1-\lambda_{1}\right) \theta\right)\right] \\
& +r^{\lambda_{2}+1}\left[A_{a} \sin \left(\left(\lambda_{2}+1\right) \theta\right)+B_{a} \sin \left(\left(1-\lambda_{2}\right) \theta\right)\right]
\end{aligned}
$$

Dealing with a sharp V- notch, the stress distributions type symmetrically in relation to the bisector angle (in mode I) can be expressed as follows (Williams, 1952; Lazzarin and Tovo, 1996; 1998):

$$
\begin{aligned}
& \left\{\begin{array}{l}
\sigma_{\theta \theta} \\
\sigma_{r r} \\
\tau_{r \theta}
\end{array}\right\}=\frac{1}{\sqrt{2 \pi}} \frac{r^{\lambda_{1}-1} \cdot K_{1}}{\left(1+\lambda_{1}\right)+\chi_{1}\left(1-\lambda_{1}\right)} \\
& \times\left[\left\{\begin{array}{l}
\left(1+\lambda_{1}\right) \cos \left(1-\lambda_{1}\right) \theta \\
\left(3+\lambda_{1}\right) \cos \left(1-\lambda_{1}\right) \theta \\
\left(1+\lambda_{1}\right) \sin \left(1-\lambda_{1}\right) \theta
\end{array}\right\}+\chi_{1}\left(1-\lambda_{1}\right)\left\{\begin{array}{l}
\cos \left(1+\lambda_{1}\right) \theta \\
-\cos \left(1+\lambda_{1}\right) \theta \\
\sin \left(1+\lambda_{1}\right) \theta
\end{array}\right\}\right]
\end{aligned}
$$

The skew-symmetric stress distributions (Mode II) are:

$$
\begin{aligned}
& \left\{\begin{array}{l}
\sigma_{\theta \theta} \\
\sigma_{r r} \\
\tau_{r \theta}
\end{array}\right\}=\frac{1}{\sqrt{2 \pi}} \frac{r^{\lambda_{2}-1} \cdot K_{2}}{\left(1-\lambda_{2}\right)+\chi_{2}\left(1+\lambda_{2}\right)} \\
& \left.\times\left[\begin{array}{l}
-\left(1+\lambda_{2}\right) \sin \left(1-\lambda_{2}\right) \theta \\
-\left(3-\lambda_{2}\right) \sin \left(1-\lambda_{2}\right) \theta \\
\left(1-\lambda_{2}\right) \cos \left(1-\lambda_{2}\right) \theta
\end{array}\right\}+\chi_{2}\left(1+\lambda_{2}\right)\left\{\begin{array}{l}
-\sin \left(1+\lambda_{2}\right) \theta \\
\sin \left(1+\lambda_{2}\right) \theta \\
\cos \left(1+\lambda_{2}\right) \theta
\end{array}\right\}\right]
\end{aligned}
$$

Parameters $K_{1}$ and $K_{2}$ are the well known Notch Stress Intensity Factors (NSIFs) related to Mode I and Mode II. $\lambda_{1}$ and $\lambda_{2}$ are mode I and mode II eigenvalues (Williams, 1952) and, finally, $\chi_{1}$ and $\chi_{2}$ are functions of the notch opening angle (Williams, 1952; Lazzarin and Tovo, 1996; 1998).

Subsequently, the stress field intensities can be quantified by the corresponding NSIFs (Gross and Mandelson, 1972):

$$
\left\{\begin{array}{l}
K_{1}(z)=\lim _{r \rightarrow 0} \sqrt{2 \pi} r^{1-\lambda_{1}} \sigma_{\theta \theta}(\theta=0) \\
K_{2}(z)=\lim _{r \rightarrow 0} \sqrt{2 \pi} r^{1-\lambda_{2}} \tau_{r \theta}(\theta=0)
\end{array}\right.
$$

Due to the shape of $3 \mathrm{~d}$ problems, in addition to the in-plane stresses, the out-of-plane stress components $\tau_{z r}$ and $\tau_{z \theta}$ can be derived by employing the following shape for $w$ function (Seweryn and Molski, 1996):

$$
w=D_{s} r^{\lambda 3, s} \cos \left(\lambda_{3, s} \theta\right)+D_{a} r^{\lambda 3, a} \sin \left(\lambda_{3, a \theta}\right)
$$

where, $\lambda_{3, s}=2 \lambda_{3, a}=\pi / \gamma$. Accordingly, anti-plane mode III shear stresses near the notch tip can be expressed as follows:

$$
\left\{\begin{array}{l}
\tau_{z r}=\frac{K_{3}(z) r^{\lambda_{3, a}-1}}{\sqrt{2 \pi}} \sin \left(\lambda_{3, a} \theta\right) \\
\tau_{z \theta}=\frac{K_{3}(z) r^{\lambda_{3, a}-1}}{\sqrt{2 \pi}} \cos \left(\lambda_{3, a} \theta\right)
\end{array}\right.
$$

and:

$$
K_{3}(z)=\lim _{r \rightarrow 0} \sqrt{2 \pi} r^{1-\lambda_{3, a}}(\theta=0)
$$

where $K_{3}(z)$ is the mode III notch stress intensity factor. Equation 15 is the extension to mode $O$ of Gross and Mandelson's definitions (Gross and Mandelson, 1972) provided for $2 \mathrm{~d}$ cases; see Equation $12 \mathrm{a}$ and $12 \mathrm{~b}$.

\section{Case of Tension Loading}

With the aim to compare the analytical frame (Lazzarin and Zappalorto, 2012), an accurate FE analysis on the model shown in Fig. 2 under tension loading has been performed. The remote stress $\sigma_{n}=100 \mathrm{MPa}$ is applied on the gross section of the plate. The ANSYS software is employed to make the Finite Element Analyses (FEA). Material is assumed to be the case of an isotropic and linear elastic with a module of the Young, E $=206000 \mathrm{MPa}$ and the Poisson's ratio $v=0.3$. A very refined and regular mesh is constructed as shown in Fig. 2.

The three stress components $\sigma_{\theta \theta}, \tau_{\mathrm{r} \theta}$ and $\tau_{\mathrm{z} \theta}$ along the notch bisector line and close to the notch tip (corner A in Fig. 2) have been plotted in Fig. 3. All the stress components have been evaluated on the plane $0.5 \mathrm{~mm}$ far from the external free surface of the $3 \mathrm{~d}$ plate. It can be observed from Fig. 3 that the value of $\sigma_{\theta \theta}$ is predominant with respect to the other two stress components. This proves the dominance of mode I with respect to other modes in the considered case.

From Fig. 4, it can be noted that the value of $K_{3}$ is lower than the other two NSIFs. This specific mode is expected to increase as the plate thickness increases (Berto et al., 2011). It is evident the linear change of $K_{3}$ through the thickness of the plate up to the maximum value, which is located in the vicinity of the free surface. In parallel, the variability of in-plane NSIFs $\left(K_{1}\right.$ and $\left.K_{2}\right)$ is shown: $K_{2}$ is constant on the major part of the plate thickness, whereas the variation of $K_{l}$ is more distributed through the thickness of the plate.

\section{Torsion Loading}

The plate with inclined diamond hole with the same material properties and element type as of the tension loading is also modelled by applying mode III loading.

The applied shear stress has been set by using the expression $\tau_{n}=3 \mathrm{~F} / \mathrm{t}^{2}$, valid for rectangular sections. It induces a nominal stress $\tau_{n}=100 \mathrm{MPa}$ on the remote section of the plate (Fig. 5). 


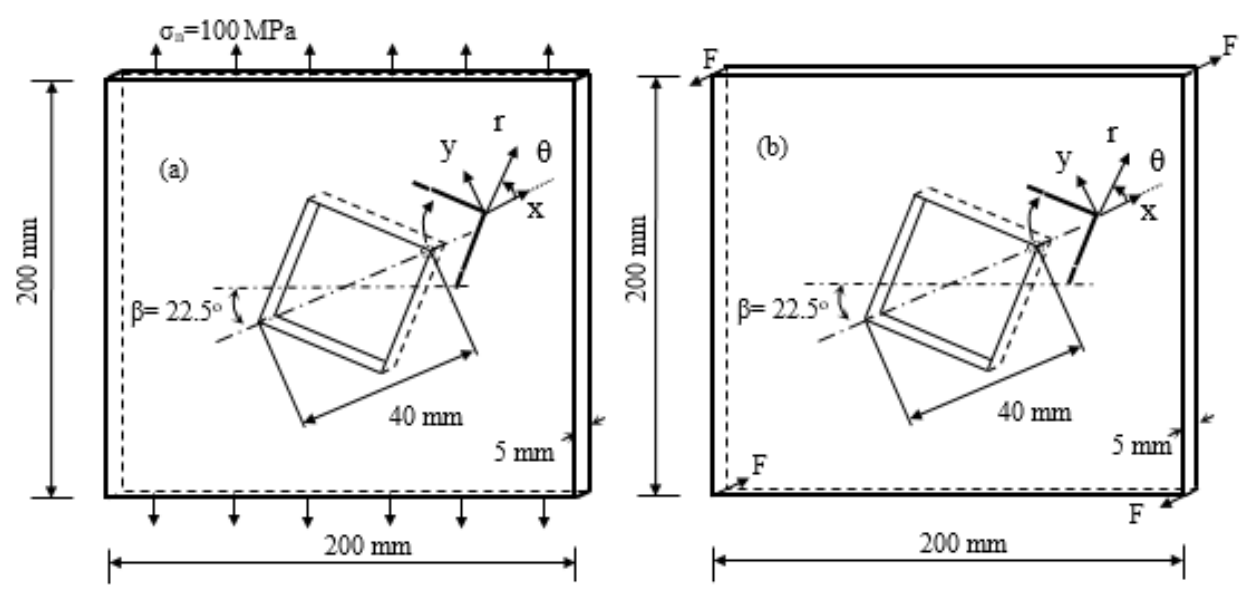

Fig. 1. Finite thickness plate weakened by inclined diamond hole under tension (a) and torsion (b)

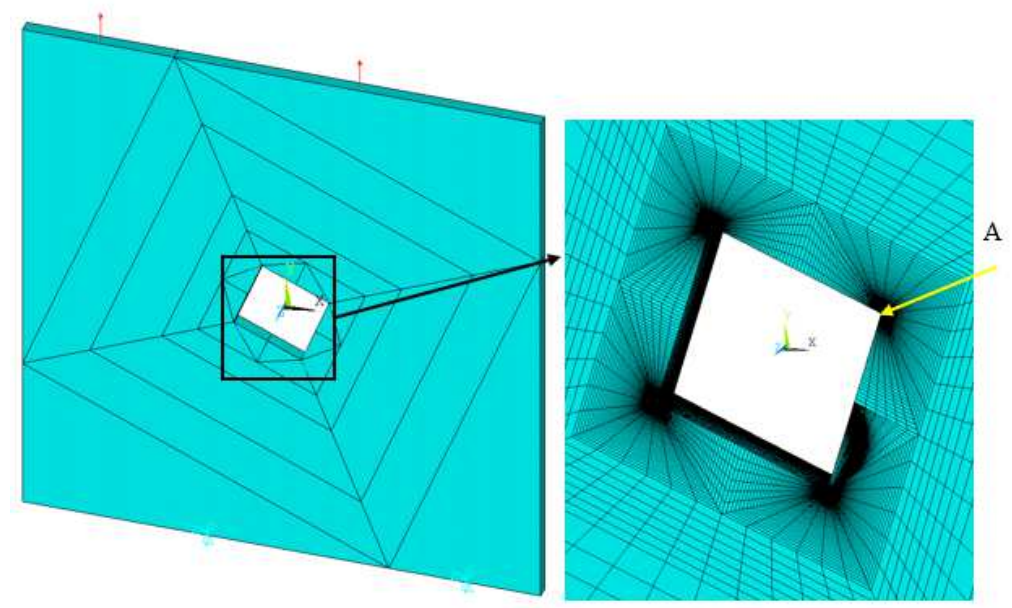

Fig. 2. Mesh pattern used for stress analysis of finite thickness plate weakened by inclined diamond hole under tension

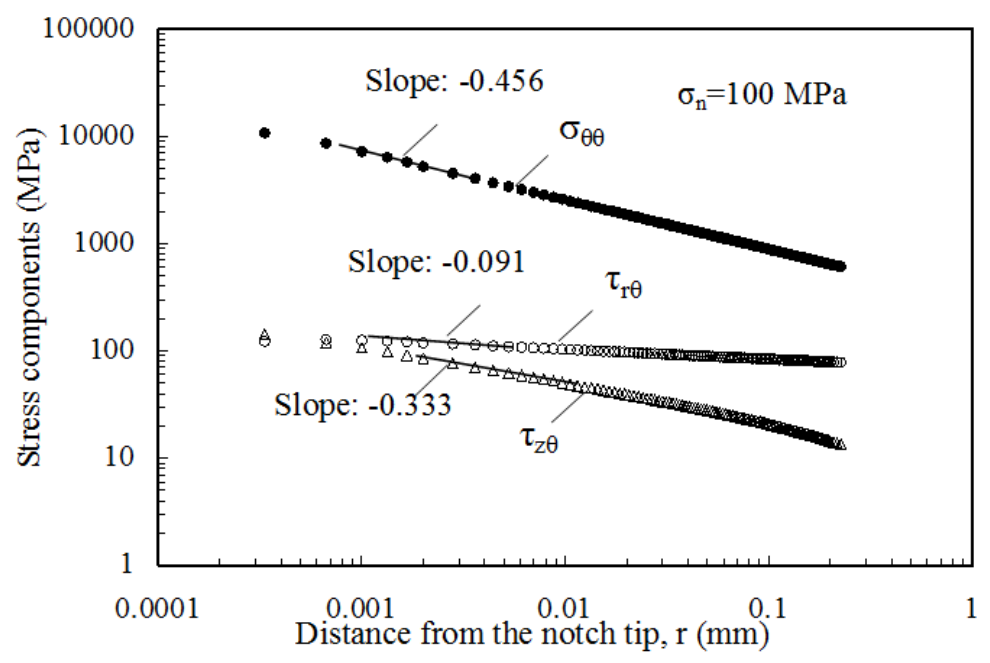

Fig. 3. Stress components $\sigma_{\theta \theta}, \tau_{r \theta}$ and $\tau_{z \theta}$ along the notch bisector line of an inclined diamond hole in a thick plate under tension. Distance from the free surface $\mathrm{z}=0.5 \mathrm{~mm}$ 


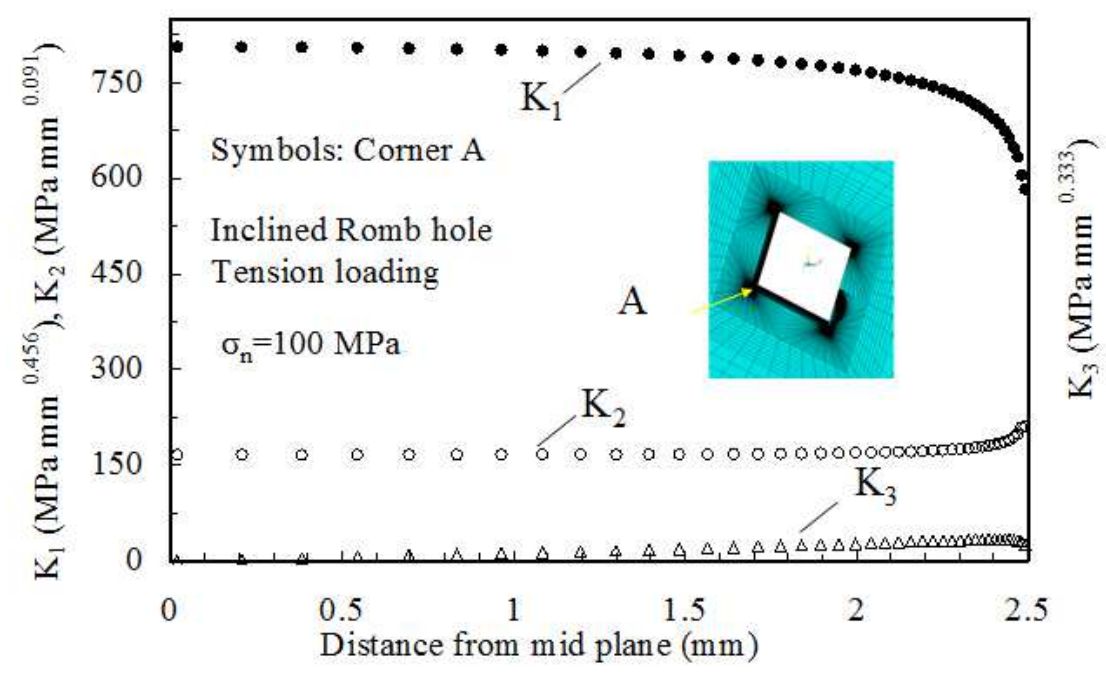

Fig. 4. Plots of NSIFs along the thickness of the plate with inclined diamond hole under tension

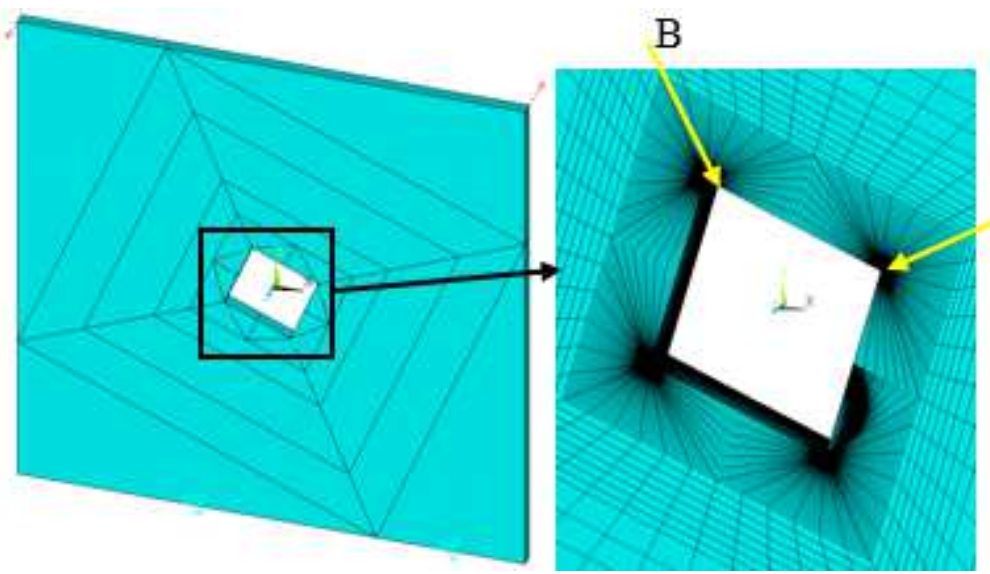

A

Fig. 5. The mesh pattern used for analysis of finite thickness plate weakened by inclined diamond hole under torsion

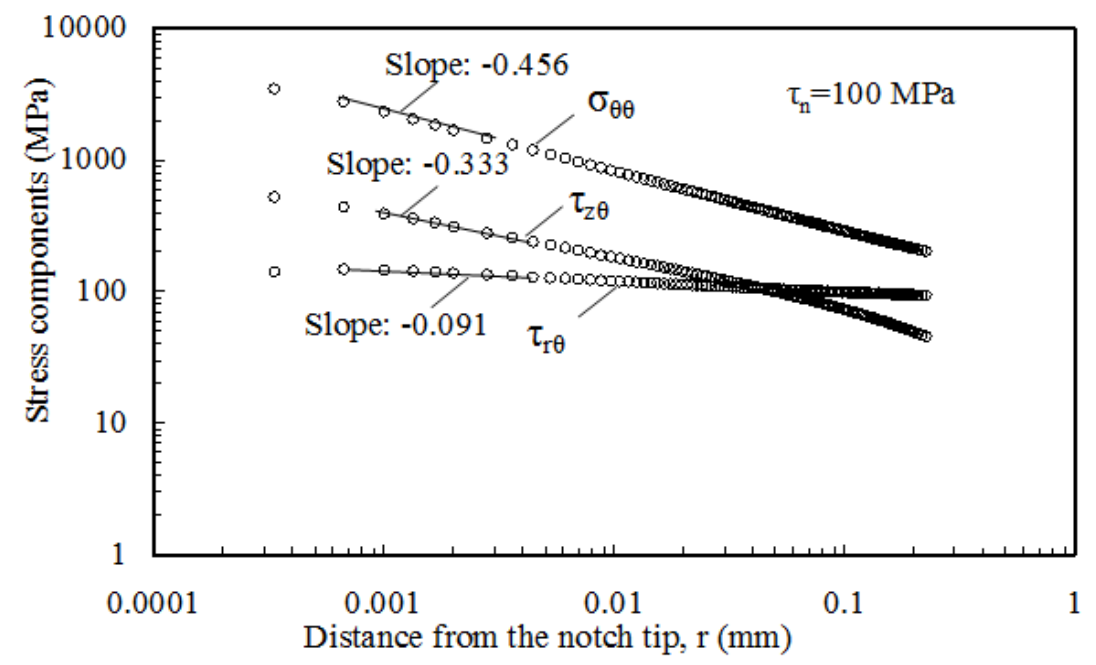

Fig. 6. Stress components $\sigma_{\theta \theta}, \tau_{r \theta}$ and $\tau_{z \theta}$ along the notch bisector line of an inclined diamond hole in a plate under torsion. Distance from the free surface $\mathrm{z}=0.5 \mathrm{~mm}$ 


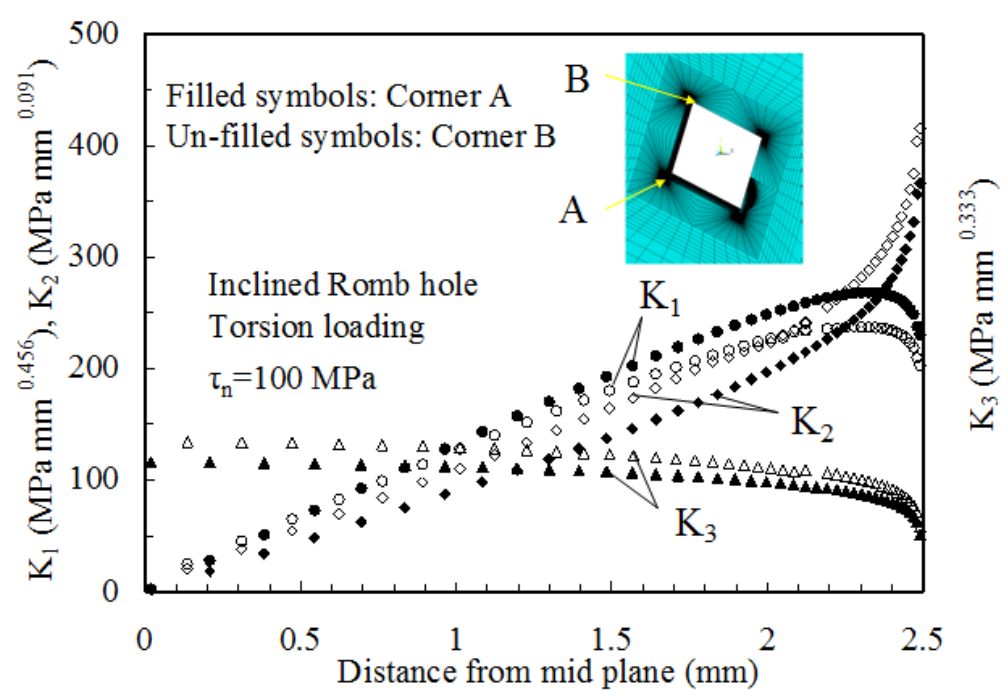

Fig. 7. Plots of NSIFs along the thickness of the plate with inclined diamond hole under torsion

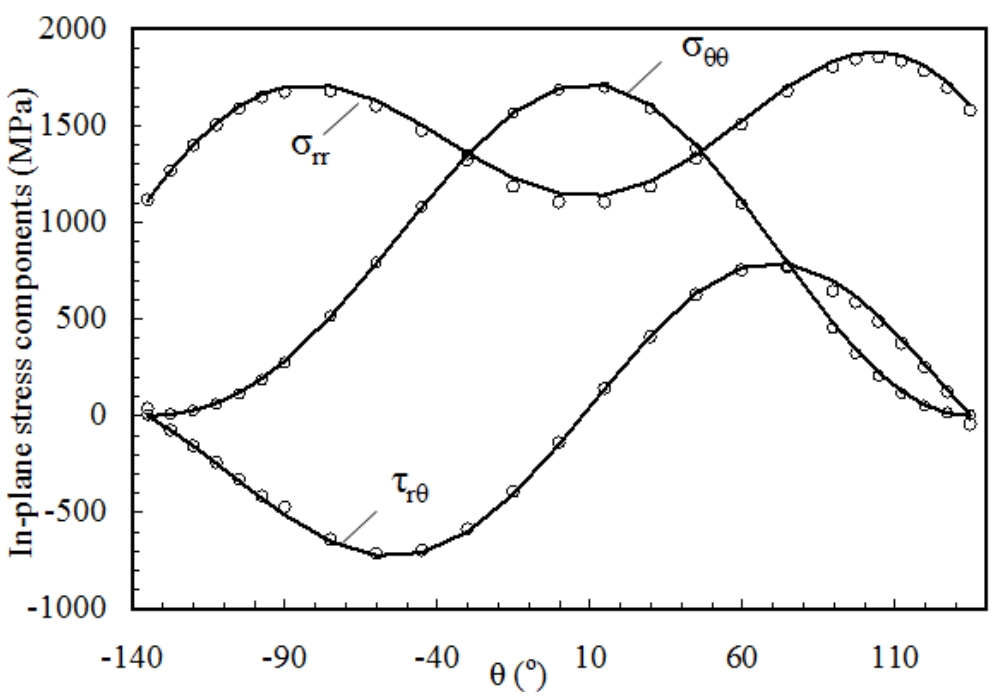

Fig. 8. In-plane stress components $\left(\sigma_{\theta \theta}, \sigma_{r r}\right.$ and $\left.\tau_{r \theta}\right)$ obtained on a circular path with radius $r=0.002 \mathrm{~mm}$ centered at the notch tip (corner A) and comparison with the theoretical predictions (Lazzarin and Zappalorto, 2012). Inclined diamond hole in a plate under torsion $\left(\tau_{n}=100 \mathrm{MPa}\right)$. Distance from the free surface $\mathrm{z}=0.5 \mathrm{~mm}$

The variation of the stress components $\sigma_{\theta \theta}, \tau_{r \theta}$ and $\tau_{z \theta}$ along the notch bisector line and close to the notch tip (corner A) are plotted in Fig. 6. Again the stresses refer to a plane at $0.5 \mathrm{~mm}$ far from the lateral surface of the $3 \mathrm{~d}$ plate.

It can be easily observed from Fig. 6 that $\sigma_{\theta \theta}$ is again higher than the $\tau_{\mathrm{z} \theta}$ corresponding to the applied mode III. Corners A and B are considered for NSIFs evaluation through the thickness of the plate, as it is shown in Fig. 7. Figure 7 shows the linear variation of $K_{1}$ and $K_{2}$ along the thickness of the plate and a parabolic trend of $K_{3}$. It was shown in (Zappalorto and Lazzarin, 2013) that the Equation system (8a-b) is valid even when the displacement field is no longer according to the Kane-Mindlin hypothesis, i.e.,
Equation 1, but is also when given in the following more general form.

$$
\begin{aligned}
& u_{x}=f^{\prime}(z) \times u(x, y) u_{y}=f^{\prime}(z) \times v(x, y) \\
& u_{z}=f(z) \times w(x, y)
\end{aligned}
$$

where, $f(\mathrm{z})$ can to be considered as a polynomial function generic of the order $n$ :

$$
f(z)=a_{0}+a_{1} z+a_{2} z^{2}+\ldots+a_{n} z^{n}
$$

It is noteworthy that Equation 17 is very similar to the equation proposed in (Hartranft and Sih, 1970) for the crack case. 


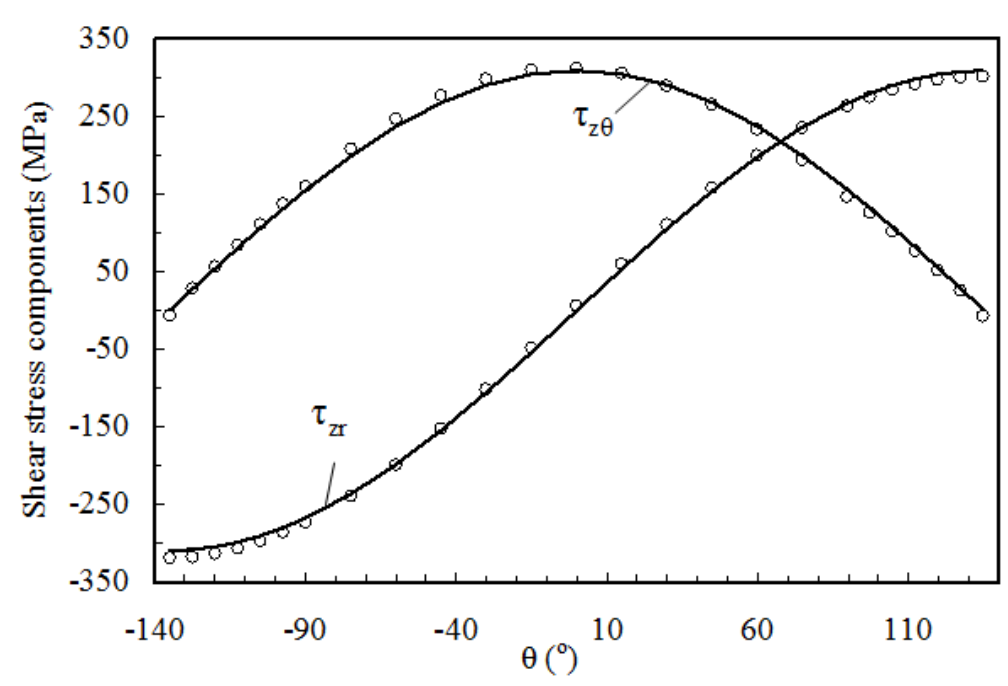

Fig. 9. Anti-plane shear stresses $\left(\tau_{z r}\right.$ and $\tau_{z \theta}$ ) obtained on a circular path with radius $r=0.002 \mathrm{~mm}$ centered at the notch tip (corner A) and comparison with the theoretical predictions (Lazzarin and Zappalorto, 2012). Inclined diamond hole in a plate under torsion $\left(\tau_{n}=100 \mathrm{MPa}\right)$

Apart from very close to the surface location (i.e., $\mathrm{z}>2.25$ from mid-plane), where corner point singularity may exist (Pook, 2003; 2000; 1992; Berto et al., 2011), on a plane at $z=0.5 \mathrm{~mm}$ from the free surface some differences between the NSIFs values may be seen at the two A and B corners. Taking into account the corner $\mathrm{A}$, the mode I seems to be the dominant mode of fracture and $K_{2}$ has a relatively lower value (about 25\% lower than $K_{I}$ ) at $z=0.5 \mathrm{~mm}$ from the free surface. On the other hand, for corner B, both $K_{1}$ and $K_{2}$ are dominant and have an equal value at the same distance from the free surface. For the both corners, the III mode adds only an effect negligible having a relatively higher value at corner B.

In-plane stresses $\left(\sigma_{\theta \theta}, \sigma_{r r}\right.$ and $\left.\tau_{r \theta}\right)$ as well as anti-plane shear stresses $\left(\tau_{z r}\right.$ and $\left.\tau_{z \theta}\right)$ obtained from FEA on a circular path with radius $r=0.002[\mathrm{~mm}]$ centered at the tip of the notch corner (A), in comparison with the theoretical prediction according to Equation 10, 11 and 14, are reported in Fig. 8 and 9.

As it can be seen from Fig. 8 and 9 a very sound it is noted an agreement between results numerical and analytical predictions.

\section{Applications}

\section{Weapons and Army}

The US Army Special Operations Command builds "eight mobile factories" that can fit into standard transport containers. These plants are based on a successful experience, the MPH. This type of "micro plants" is the culmination of the factory idea, with threedimensional printing technologies.
According to the US military, three-dimensional printing reduces production costs by $97 \%$ and production time by $83 \%$.

Texas student Cody Wilson managed to manufacture a firearm using a 3D printer. If the bulk of the weapon is made of molded plastic, manufactured using the 3D printer, the barrel and butt remain metal. Once demonstrating the effectiveness of the weapon, the creator of that gun, then shared plans to manufacture the weapon on the Internet. Originally, the creator of this firearm wished to be able to fire at least twenty balls with the weapon thus created. He could only fire six, before the weapon completely disintegrated.

\section{Aeronautics}

EADS, the parent company of Airbus, has plans to produce all parts of aircraft using ALM-enabled (additive layer manufacturing) technology. Airbus already produces parts of these aircraft thanks to the $3 \mathrm{D}$ printing process especially for the A350 XWB. What is valuable for aeronautics is 30 to $55 \%$ lighter parts compared to traditional and handmade products. The A350 already adopts more than 1,000 pieces manufactured in this way.

In 2014 SpaceX succeeded in replacing certain (metallic) components of their rockets with 3D printed parts.

\section{Medicine and Research}

The field of medicine also benefits from 3D printing, with the creation of a material similar to a bone or the creation of prostheses and implants (artificial hips, dental and personalized hearing aids) and customized exoskeletons. Recently researchers at AECS (University 
of Wollongong) have designed a pencil, the BioPen, which can print stem cells (nerve, muscle, bone) on injured areas.

Three-dimensional printing makes it possible to materialize hollow spaces or soft organs.

Authorized in 2015 by the FDA, the first 3D drug is marketed in the United States in April 2016 by Aprecia, which is exclusive to the pharmaceutical industry for a patented technique by MIT. The active substance is levetiracetam. The pill which are more porous thanks to 3D printing dissolves more quickly, facilitating its ingestion by people with dysphagia or swallowing disorders.

A humanoid robot and a cheap bionic hand, Bionicohand, were created from 3D printing.

\section{Industry}

In industry there is today a huge amount of $3 \mathrm{D}$ printing applications.

Various types of parts are produced using this new technique.

\section{Discussion}

Three dimensions or three-dimensional or 3D are expressions that characterize the space around us, as perceived by our vision, in terms of width, height and depth. The term "3D" is also (and improperly) used to refer to the representation in synthetic (digital) images, the relief of stereoscopic images or other images in relief and sometimes even the simple stereophonic effect, which Can only render 2D (it is therefore only the calculation of the projected projections, the shadows, the renderings of materials). In mathematics, this notion corresponds to Euclidean geometry in space.

The space is marked by three orthogonal axes, contrary to the plane composed of two dimensions. Since its origins and long before the appearance of color, cinema has exploited stereoscopy, mainly through the processes of anaglyphs.

During the $1950 \mathrm{~s}$, the Hollywood studios exploited the polarized filter system and then, from the 2000 s onwards, with the adoption of digital formats, the rooms also equipped the electronic principle with alternating shutter ("active" glasses).

Despite some anaglyphic experiments since the $1930 \mathrm{~s}$ and $1950 \mathrm{~s}$, the commercial success of 3D television became a reality from the end of the $1990 \mathrm{~s}$ to a real industrial development from the year 2000. Two principles and devices for Stereoscopic effects are commercialized: Of the "active" type with electronic bezels or of the "passive" type with polarized filter goggles. More complex and costly to industrialize in particular with regard to large screens, autostereoscopy (relief effect without bezel) was introduced with Alioscopy since the late 1990 s, on the lenticular principle. Stereoscopic videoprojection intended for the general public requires electronic "active" glasses or a special screen (metallized or offering some refraction of light) associated with "passive" glasses with polarized filters.

Three-Dimensional (3D) effects close to sharp corners of a hole in a plate with finite thickness are investigated in the present contribution. The results from detailed 3D finite element model are analyzed to investigate the stress intensity of various fracture modes caused by the presence of a finite thickness. The results expressed in terms of stressed are compared with some recent equations. The comparison between numerical and theoretical results shows a sound agreement.

The synthesis of three-dimensional images, often abbreviated 3D (3D for three dimensions: $x, y, z$, the three axes which constitute the orthonormal reference frame of geometry in space) is a set of techniques derived in particular from CAD Representation of objects in perspective on a computer monitor. It is currently used extensively in digital art in the film industry, initiated by the Pixar, Disney, DreamWorks, Blue Sky, Illumination and ILM studios and, since 1992, in many video games. It should not be confused with 3D terms relating to relief or stereoscopy.

The difference is that before the appearance of computers, perspective was obtained by graphical methods derived directly from projective geometry. Currently, the drawings are computed numerically from three-dimensional numerical data. This makes it easy to change the projection center and its various parameters. In particular, it is possible to calculate series of projections by displacing the projection point and thus performing animations.

Today's computer-generated images are based on the same principles of projection on a plane and are inseparable from the history of computer science. Synthetic images began in the early 1950 s in the United States and were reserved for research, including academic research. A system consisting of a cathode ray tube and an optical pencil was developed, according to an idea by Ivan Sutherland of the Massachusetts Institute of Technology (MIT), for aerial control of the Air Force. 1961 a cross was added on the screen to indicate the position of the optical pencil. We then practiced the 2D image, then the 3D image, more expensive in computing time and financially.

The universities also used three-dimensional images and in 1967 the University of Utah specialized in this field, especially Professors David C. Evans and Ivan Sutherland, who tried to model various objects such as the car Ivan Sutherland and who in 1968 founded the company Evans \& Sutherland. Then in 1970, Xerox created the PARC (Palo Alto Research Center), which will work very freely, because without commercial 
objectives. It will come out many discoveries that Xerox will not exploit. In 1975 was created one of the most famous images of computer graphics, the teapot now a classic test object for 3D applications. The teapot that served as a model now rests at the Boston Computer Museum, near a computer that reproduces its threedimensional image.

Until the $1980 \mathrm{~s}$, few people approached this area because of material costs. But the advent of personal computers, such as the Xerox Star, the IBM-PC in 1981 and the Apple Macintosh in 1984, democratized the use of $3 \mathrm{D}$ for study and production. Technology results in superb simulations of shuttles or rockets by NASA, or landscapes and faces.

But it was in the $1990 \mathrm{~s}$ that the synthetic image and 3D became more democratic and developed, including the arrival of more powerful real-time hardware such as 3D maps at Silicon Graphics on High-end workstations then later in Amiga and PC computers with 3D maps like 3DFX or in game consoles like PlayStation, Dreamcast. Since then, 3D acceleration is an integral part of computer hardware.

The 3D image synthesis consists essentially of two stages:

- Model what you want to visualize or represent. This step is called modelling

- Perform visualization of what you modelled. This step is called rendering

In each of these steps, a large number of techniques exist. Although theoretically the techniques of modelling and rendering are independent, it goes without saying that the rendering must be able to exploit the data modelled and very often a technique of modelling is closely associated with a technique of rendering.

The modelling will consist in having the computer store a set of geometric data and graphic characteristics allowing to represent the model. This model is usually called 3D scene, according to English scene which means "view", we will say then "3D view".

Three-dimensional modelling is the step in threedimensional computer graphics, which consists in creating, in a 3D modelling software, an object in three dimensions, by adding, subtracting and modifying its constituents.

Polygonal modelling: The model is assimilated to a set of polygons: This polyhedron is therefore described by the list of vertices and edges. If the polygons are oriented, the exterior and the interior of the model can be differentiated. If we wish to remove the ambiguity brought by non-planar polygons, we will use only triangles: this triangulation can be forced by the modeller.

Without smoothing effect, the object will appear angular if the facet definition is weak. In general, the smoothing of Gouraud, realized by the 3D graphics card, is sufficient to give a smoother aspect to the model.
Polygon modelling uses the same basic tools on all 3D software, as well as some specific tools for generic software, specialized software and plug-ins and scripts.

Among these tools, the most common are extrusion, cutting (cut, split ...), weld/collapse and revolution.

The extrusion consists of raising one or more faces (adjacent or not) or a 2D profile along a trajectory and creating the faces that fill the vacuum caused by the displacement of the starting element. For example, extruding a circle gives an open cylinder or pipe that follows the trajectory.

The cut consists of creating edges on a mesh, as well as the points corresponding to the intersections of the existing and newly created edges. One can thus refine a model, by adding details on certain regions, in particular by the addition of edges loops (edgeloops) extremely used in organic modelling.

The welding/retraction consists in welding the vertices (vertices) to each other, to simplify a mesh, to block holes or to converge edges.

The revolution consists in turning a $2 \mathrm{D}$ profile around a 3D axis: A volume of revolution is obtained.

This is the technique mostly used in video games and animation. The polygonal modeling induces a margin of error of proportions and dimensions mostly invisible to the naked eye. In animated cinema, organic 3D models are most often smoothed. The smoothing consists in subdividing a mesh (an iteration corresponds to a subdivision of each edge, in the case of the face with four sides, a subdivision in four faces) and rounding the faces obtained according to different algorithms, in order to erase the angular effect Of models obtained by polygonal modelling.

Modelling methods from examples allow reuse of existing models and build an existing model by assembly. Unlike solid geometry, primitives are not simple geometric objects (cone, cylinder, cube, etc.), but are sub-parts of a polyhedron. The end result is therefore a polyhedron and the surface thus obtained may be a variety, or not.

Modelling methods using examples use search algorithms within a 3D model database and mesh assembly1. Two types of use may be considered:

- Suggestion of segments to add to a model being created

- Replacing an existing part with another geometrically or functionally similar

The search mechanisms in the database can be made from keywords, 2D sketches or 3D meshes.

\section{Curve Modeling (NURBS)}

The Non-Uniform Basic Rational Basic Spline (NURBS) model consists of a network of curves created by control vertices. The interpolation of the curves 
between these points can be done automatically according to a NURBS algorithm, by manipulation of Bézier curve tangents, or by modification of the interpolation parameters.

Curve modelling is based on an adaptive mesh, designed to adapt its subdivisions to the complexity of the curves in a given region. A perfectly flat surface will have a very low or no subdivision number. This is the most accurate modelling technique. This is why it is used extensively in architecture and industrial CAD, where precision is paramount (especially when 3D models serve as a reference for machine tools).

\section{Surface Subdivision Modelling}

This method regroups a little of the two conventional methods (polygonal and NURBS). It consists in accelerating the process by automatically subdividing part of the surface. This allows you to add details to certain places only, regardless of the number of faces included in the overallity of the object.

It approximates the polygonal modelling by the techniques used during the creation of the shape and the modelling by NURBS with respect to the surface rendering, that is to say its roundness.

It is present in many professional software (Blender, Maya, 3ds Max, Lightwave, Softimage, Modo ...).

\section{Parametric Modelling}

Parametric modelling is the preferred mode of operation of current industrial CAD software. This is to define an entity by parameters that can be easily changed. In this way, the definition of the part is easily changed.

\section{Some 3D Modelling Software}

3D Turbo
3DReshaper
Art of Illusion
Autodesk Software
3D Studio Max
Alias Products
$\quad$ Maya
$\quad$ XSI
Mudbox
blender
Cinema 4D
Daz3D
Dassault Systèmes Software
$\quad$ SolidWorks
CATIA
CADDS
FreeCAD
FormZ
Hexagon
HyperMesh
Intericad

\author{
Lightwave \\ Modo \\ Pro/Engineer \\ Rhinoceros 3D \\ Trimble Software \\ Sketchup \\ Tekla Structures \\ Solid Edge ST \\ Wings 3D \\ ZBrush \\ PCon.planner
}

\section{Conclusion}

In this study an attempt is made to examine the proposed theory in (Lazzarin and Zappalorto, 2012) by investigating the stress fields in the one diamond hole sharp corners vicinity for a plate with finite thickness under tension and twisting loading conditions. The FE results have confirmed the presence of coupled modes at the V-notch tip, showing a good agreement with the inplane and out-of-plane theoretical stress distributions.

\section{Acknowledgement}

This text was acknowledged and appreciated by Professor Guanying Chen Harbin Institute of Technology and SUNY Buffalo China, Associate Professor Aniello Riccio SECONDA UNIVERSITA' DEGLI STUDI DI NAPOLI Italy, Dr. (Ms.) Shweta Agarwala Senior Research Scientist at Singapore Center for 3D Printing Nanyang Technological University Singapore, whom we thanks and in this way.

\section{Author's Contributions}

All the authors contributed equally to prepare, develop and carry out this manuscript.

\section{Ethics}

This article is original. Authors declare that are not ethical issues that may arise after the publication of this manuscript.

\section{References}

Berto, F., P. Lazzarin, A. Kotousov and S. Harding, 2011. Out-of-plane singular stress fields in Vnotched plates and welded lap joints induced by inplane shear load conditions. Fatigue Fracture Eng. Mater. Struct., 34: 291-304.

DOI: $10.1111 /$ j.1460-2695.2010.01518.x

Gross, R. and A. Mendelson, 1972. Plane elastostatic analysis of $\mathrm{V}$-notched plates. Int. J. Fracture Mechan., 8: 267-276. DOI: 10.1007/BF00186126 
Hartranft, R.J. and G.C. Sih, 1970. An approximate three-dimensional theory of plates with application to crack problems. Int. J. Eng. Sci., 8: 711-729. DOI: 10.1016/0020-7225(70)90054-6

Heyder, M., K. Kolk and G. Kuhn, 2005. Numerical and experimental investigations of the influence of corner singularities on 3D fatigue crack propagation. Eng. Fracture Mechan., 72: 2095-2105. DOI: 10.1016/j.engfracmech.2005.01.006

Jin, Z.H. and R.C. Batra, 1997. A crack at the interface between a Kane-Mindlin plate and a rigid substrate. Eng. Fract. Mech., 57: 343-354. DOI: 10.1016/S0013-7944(97)00036-2

Kane, T.R. and R.D. Mindlin, 1956. High-frequency extensional vibrations of plates. J. Applied Mechan. Trans.

Kotousov, A., 2005. On stress singularities at angular corners of plates of arbitrary thickness under tension. Int. J. Fracture, 132: L29-L36. DOI: $10.1007 / \mathrm{s} 10704-005-4481-y$

Kotousov, A., 2007. Fracture in plates of finite thickness. Int. J. Solids Struct., 44: 8259-8273. DOI: 10.1016/j.ijsolstr.2007.06.011

Lazzarin, P. and M. Zappalorto, 2012. A threedimensional stress field solution for pointed and sharply radiused $\mathrm{V}$-notches in plates of finite thickness. Fatigue Fracture Eng. Mater. Struct., 35: 1105-1119.

DOI: 10.1111/j.1460-2695.2012.01698.x

Lazzarin, P. and R. Tovo, 1996. A unified approach to the evaluation of linear elastic stress fields in the neighborhood of cracks and notches. Int. J. Fracture, 78: 3-19. DOI: 10.1007/BF00018497
Lazzarin, P. and R. Tovo, 1998. A notch intensity factor approach to the stress analysis of welds. Fatigue Fracture Eng. Mater. Struct., 21: 1089-1103. DOI: 10.1046/j.1460-2695.1998.00097.x

Nakamura, T. and D.M. Parks, 1989. Antisymmetrical 3D stress field near the crack front of a thin elastic plate. Int. J. Solids Struct., 25: 1411-1426. DOI: 10.1016/0020-7683(89)90109-1

Pook, L.P., 1992. A note on corner point singularities. Int. J. Fatigue, 53: 3-8.

Pook, L.P., 2000. Finite element analysis of corner point displacements and stress intensity factors for narrow notches in square sheets and plates. Fatigue Fracture Eng. Mater. Struct., 23: 979-992. DOI: 10.1046/j.1460-2695.2000.00358.x

Pook, L.P., 2003. A finite element analysis of cracked square plates and bars under antiplane loading. Fatigue Fracture f Eng. Mater. Struct., 26: 533-541. DOI: $10.1046 /$ j.1460-2695.2003.00618.x

Seweryn, A. and K. Molski, 1996. Elastic stress singularities and corresponding generalized stress intensity factors for angular corners under various boundary conditions. Eng. Fracture Mechan., 55: 529-556. DOI: 10.1016/S0013-7944(96)00035-5

Williams, M.L., 1952. Stress singularities resulting from various boundary conditions in angular corners of plates in extension. J. Applied Mech., 19: 526-528.

Zappalorto, M. and P. Lazzarin, 2013. Threedimensional elastic stress fields ahead of notches in thick plates under various loading conditions. Eng. Fracture Mechan., 108: 75-88.

DOI: 10.1016/j.engfracmech.2013.02.031 